MARCIN MUSIAE

Uniwersytet Wrocławski

E-Mail: musial.wroclaw@gmail.com

\title{
GRENZEN UND BEDEUTUNG DES SAKRALRAUMS DER FRANZISKANER- REFORMATEN IM FRÜHNEUZEITLICHEN STADTRAUM IN SCHLESIEN
}

\section{DER FRANZISKANISCHE RAUM ALS ELEMENT DES SAKRALRAUMS}

Der franziskanische Raum ist vor allem als ein Element des Sakralraums zu betrachten, d. i. als eine gewisse Erscheinung, die einem Verschlüsselungsprozess mithilfe von Markern, Zeichen und Symbolen unterliegt, welche als ständige bzw. zeitliche Elemente des wirklichen Raums gelten ${ }^{1}$. Die Sakralisierung des Raums ist somit an die kollektiven Bedeutungen angelehnt, die ihm zugeschrieben ${ }^{2}$ und vom Empfänger eindeutig entschlüsselt und interpretiert werden können. $\mathrm{Zu}$ solchen Elementen des Raums gehören daher die ihn mitgestaltenden Gebäude, Orte und die mit ihnen verbundenen Bedeutungen. Im Prozess der Raumprägung spielt selbstverständlich die Architektur eine

1 E. Klima, Przestrzeń religijna miasta, (2011), S. 17; B. Jałowiecki, Czytanie przestrzeni, (2012), S. 9-71; ders., Społeczne wytwarzanie przestrzeni, (2010), S. 19-36.

2 M. Kowalewski, D. Kowalewska, Katedra jako stereotyp przestrzenny. Przypadek kościoła pw. św. Jakuba w Szczecinie, in: Miasto i sacrum, hg. v. M. Kowalewski, A. M. Królikowska, (2011), S. 75. 
wesentliche Rolle. Einerseits ist sie ein materielles Element des Raums, ein geschlossener Raum, der bestimmte soziale Funktionen erfüllt. Andererseits kann man die Architektur auch als einen Bedeutungsträger begreifen $^{3}$. Alle ihre Komponenten (wie z. B. Konstruktion, Funktion, Komposition, Dekoration oder Symbolik) können als Werkzeuge der Raumprägung betrachtet werden ${ }^{4}$. Es ist besonders deutlich im Falle der Sakralarchitektur ${ }^{5}$. Im Rahmen dieses Beitrages, am Beispiel des schlesischen Teiles der böhmischen St. Wenzel-Provinz der Franziskaner-Reformaten, konzentriere ich mich sowohl auf die Beziehungen zwischen einem architektonischen und einem sozialen ${ }^{6}$ Raum als auch auf zwei damit verbundene Aspekte: die Grenzen und die Bedeutung des franziskanischen Sakralraums.

In diesem Zusammenhang kann der franziskanische ${ }^{7}$ Raum auf zweierlei Weise definiert werden, was durch das Ausmaß der analysierten Erscheinung bedingt ist. Zum einen kann man nach Henryk Gapski annehmen, dass der Raum einfach „durch die Klöster der Minderen Brüder, die im Rahmen einer Provinz strukturiert sind, sowie durch eine andere territorial organisierte Ordensstruktur gestaltet wird"8. Es ist aber gleichzeitig kein Begriff, der mit dem,Klosternetz zu identifizieren ist, denn im Gegensatz dazu hebt der „franziskanische Raum“ die für die Mendikantenorden relevante Frage der Einheit der Brüder hervor, die über die Mauern eines einzelnen Klosters zugunsten aller

3 E. Niezabitowska, Ewolucja konceptu przestrzeni $w$ teorii architektury, (2008), S. 14,79 .

4 Ebenda; Jałowiecki, Społeczne, S. 23.

5 Z. B. J. J. Czechowicz, Architektura sakralna Krakowa - sztuka formowania przestrzeni, in: Sztuka tworzenia miejsc, hg. v. S. Hryń, (2008), S. 25-36.

6 Jałowiecki, Społeczne, S. 37-46.

7 Das Adjektiv „franziskanisch“ wurde in diesem Beitrag als eine abgekürzte Form vom Adjektiv, das vom Namen Franziskaner-Reformaten kommt, genutzt.

8 H. Gapski, Klasztory zakonów franciszkańskich w Europie Środkowo-Wschodniej w XVIII wieku. Studium przestrzeni franciszkańskiej, (2013), S. 7. 
Provinzhäuser hinausgeht ${ }^{9}$. Eine gewisse Visualisierung des so verstandenen franziskanischen Raums bietet die 1737 angefertigte Karte ${ }^{10}$ der böhmischen St.-Wenzel-Provinz der Franziskaner-Reformaten ${ }^{11}$. In eine differenzierte Topografie der drei Länder der Böhmischen Krone: Böhmen, Mähren und Schlesien, trug man die Umrisse von über dreißig Kirchen und Klöstern der Provinz ein und sorgte dafür, ihre Unterschiede wie auch Ähnlichkeiten zu betonen, welche beispielsweise in der Ordnung der Gebäude oder dem Verzicht auf Hochtürme zum Ausdruck kamen. Über den ganzen Raum der Reformaten wacht einerseits eine Kartusche mit einem Provinzsiegel mit dem Bildnis St. Wenzels, andererseits das Wappen des Franziskanerordens, für dessen treuesten Söhne die Reformaten gehalten werden wollten ${ }^{12}$.

9 Ebenda.

10 Die Karte befindet sich in: S. Wrbczansky, Nucleus Minoriticus, seu vera et sincera relatio originis et progressus provinciae Bohemiae, conventuum et residentiarum fratrum et sororum sancti-monialium ordin. minor. S. P. Francisci Strict. Observ. Reform. in Provincia, sub patrocinio Sancti Wenceslai ducis et martyris, Per Bohemiam, Moraviam et Silesiam existentium. Nec non fundatorum, capitulorum, commisariorum, provincialiorum aliorumque memoria dignorum, ex archivis, proto-collis et manuscriptis, sedule collectorum compilatum, (1746).

11 Der Orden der Minderen Brüder Reformaten (lat. Ordo Fratrum Minorum Strictoris Observantiae Reformatorum) hat sich seit der zweiten Hälfte des 16. Jahrhunderts im Rahmen des aus der franziskanischen Familie ausgegliederten Orden der Minderen Brüder der Strengeren Observanz entwickelt. Diese Gemeinschaft, die sich auf Verbindung strenger Einhaltung von den durch die Führung eines einsiedlerischen Lebens gekennzeichneten Armutsgelübden mit gleichzeitiger Erfüllung des Apostolates konzentrierte, hatte in Schlesien zwölf Häuser in zwei Provinzen (böhmische Provinz vom Heiligen Wenzel und kleinpolnische Unsere Liebe Frau von der Engeln-Provinz, hier die Klöster in: Gleiwitz und Sankt Annaberg). Das Objekt meiner Interessen sind zehn franziskanische Klöster der böhmischen Provinz vom Heiligen Wenzel in: Neiße (gegründet 1620), Jauer (1638), Troppau (1655-1690), Groß-Glogau (1640), Leobschütz (1666), Namslau (1675), Breslau (1678), Ratibor (1686), Liegnitz (1700 r.) und Goldberg (1704).

12 Die böhmische St.-Wenzel-Provinz der Franziskaner-Reformaten entstand 1660. 1755 wird, auf Drang des preußischen Staates der nach den schlesischen Kriegen in den Jahren 1740-1742 auch Schlesien umfasste, aus der böhmischen Provinz eine neue schlesische Provinz der Heiligen Hedwig ausgeteilt. Diese Provinz existierte bis 
Die obige Definition des Raums, die den Begriff der Einheit innerhalb einer Provinz hervorhebt, bewirkt, dass die im Rahmen einer Provinz unternommene Konsolidierungstätigkeit, welche in der Herausbildung eines einheitlichen Konstrukts der Identität aufgrund der Geschichtsschreibung oder der in der Gesetzgebung ausgedrückten Tätigkeitsnormen besteht, gleichzeitig zu jenem Element wird, das den Raum gestaltet, d. i. ihn verschlüsselt. Ein weiteres Bindeglied ist eine vereinheitlichte Visualisierungspolitik, die auf die Herausbildung einer kohärenten Visualisierungsbotschaft zurückgeht, welche die Hauptregeln und Ideen widerspiegelt, mit denen sich eine bestimmte Provinz identifiziert. $\mathrm{Zu}$ diesem Bereich gehören sowohl die Bildprogramme, die Ausstattung der Klosterkirchen wie auch die Architektur der Klostergebäude samt ihrer Stilistik und den aus der Bautradition oder der Gesetzgebung hervorgehenden Lösungen im Bereich der Disposition des Innenraums oder der angewandten Formen. Soweit der Raum im Unifizierungsprozess und im Rahmen der inneren Konsolidierung einer Provinz gestaltet wird, kann jedes der an diesem Prozess beteiligten Klöster alle für die ganze Gemeinschaft repräsentativen Eigenschaften nachweisen. Somit kann man ein einzelnes Kloster, das zur Stadtstruktur gehört und in einer bestimmten Stadt den franziskanischen Raum gestaltet, in jenen Kategorien untersuchen, die es ermöglichen, auch die ganze Provinz widerzuspiegeln. Man kann sagen, dass eine solche Perspektive auf der Mikroskala die zweite Variante dieser Frage erlaubt. Darauf möchte ich mich konzentrieren, indem ich zwei wichtige Aspekte wie folgt analysiere: die Grenzen und die Bedeutung des „kleinen“ franziskanischen Raums in Bezug auf eine schlesische Stadt in der Neuzeit.

Als Quellengrundlage in dieser Raumanalyse gilt die Chronica de origine et constitutione Provinciae Bohemiae Ordinis Fratrum Minorum

zur Säkularisation in Schlesien 1810. L. Teichmann, Ordensprovinzen der Franziskaner in Schlesien, „Archiv für Schlesische Kirchengeschichte“, 43 (1985), S. 263-274; Ch. Reisch, Die Franziskaner im heutigen Schlesien von Anfange des 17 Jahrhunderts bis zur Säkularisation, „Zeitschrift des Vereins für Geschichte Schlesiens“, 47 (1913), S. 276-300. 
S. Francisci Reformatorum ${ }^{13}$ von Bernard Sannig (1637-1704) ${ }^{14}$, einem Franziskaner-Reformaten, gebürtig aus Neiße (Nysa) in Schlesien. Eben auf diese handschriftliche Chronik aus dem Jahr 1678 gehen die neuzeitliche Geschichtsschreibung der Provinz wie auch eine fest definierte Auffassung vom offiziellen Gedächtnis einer Gemeinschaft zurück. Sie entstand nämlich zu jener Zeit, als die Provinzstrukturen nach der Reformation und dem Dreißigjährigen Krieg wiederhergestellt wurden. Es war eine Zeitspanne, in der die Reformaten darum bemüht waren, die im 16. Jahrhundert verlorenen Klöster wiederzuerlangen, und in den mit den protestantischen Stadträten oder den anderen Franziskanergemeinschaften geführten Gerichtsprozessen historisch-rechtliche Argumente brauchten, die ihre Rechte gegenüber den Ansprüchen der anderen Einrichtungen bestätigen sollten. Im Endeffekt wurde ihnen eine feste Begründung angegeben, die die Anwesenheit der Reformaten in den gewählten schlesischen, böhmischen und mährischen Städten legitimierte.

Ein wesentliches Element der Chronica de origine [...] von Bernard Sannig ist ein erweiterter Katalogteil, der alle Klöster der Provinz im Jahr 1678 darstellt. Unter den schlesischen Häusern schilderte der Autor die Klöster in Neiße, Glogau (Głogów), Namslau (Namysłów), Breslau (Wrocław), Jauer (Jawor), Troppau (Opawa) und Leobschütz (Głubczyce). Im Rahmen einer festen Beschreibungsstruktur fügt Sannig jedes Mal die Geschichte eines bestimmten Klosters hinzu, indem

13 Národní Archív Praha (weiter: NA Praha), Archiv České fratiškánské Provincié (weiter: RF), Sign. 20: B. Sannig, Chronica de origine et constitutione Provinciae Bohemiae Ordinis Fratrum Minorum S. Francisci Reformatorum, (1678). Die Exemplare der Chronica de origine von Sannig befinden sich sowohl in Praha (NA Praha, RF, Sign. 17 (Autograph), 18 (Autograph), 19 (Abschrift) und in zwei Bänden: Sign. 20, 21 (Abschrift)), als auch in Archiwum Państwowe we Wrocławiu (Zespół Rękopisów Archiwalnych Rep. 135, Sign. 373 (Autograph)).

14 Die Biographie Bernard Sannigs wurde in Teilen zwischen 1920 und 1930 publiziert: K. Minařík, Provinciál P. Bernard Sannig, učenec, spisovatel a organisátor františkánské provincie (1637-1704), „Časopis pro Katolického Duchovenstwa“, 61 (1920) - 71 (1930), s. http://depositum.cz/knihovny/ckd/ (aufgerufen: 12.6.2017). 
er zunächst an die Gestalt des Begründers oder Stifters sowie an die damit verbundene Stiftung des Grundstücks anknüpft. Den nachfolgenden Auszug aus der Geschichte der Reformaten in Schlesien trennt eine sichtbare Zäsur im zweiten Jahrzehnt des 16. Jahrhunderts. Sie bestimmt die Zeit der Reformation und der Verbannung aus dem Kloster wie auch des Übertritts der Brüder zum Luthertum bzw. des einhergehenden Aussterbens des Konvents, der angesichts der mangelnden neuen Berufungen und des Ausfallens von Almosen wenige Chancen auf Fortbestehen und Entwicklung hatte. Die Zeitspanne nach der Reformation gilt als eine Zeit, in der die Ordensbrüder darum bemüht waren, ihren alten Sitz wiederzuerlangen. Der Erfolg war durch Posaunen und den Lobgesang Te Deum laudamus am Tag der feierlichen Inthronisation der Brüder in dem ihnen restituierten Kloster verkündet worden. Besonders interessant ist ein dem historischen Teil beigefügtes Fragment, das sich auf die faktische Beschreibung eines vom Konvent benutzten Grundstücks bezieht, wo auch genaue Angaben zu dessen Lage in der Stadt mit Mauer-, Wand- und Gärtenlängen angeführt wurden. Jeder Auszug, der höchstwahrscheinlich zur selben Zeit wie die von Sannig durchgeführte Visitation der Provinz entstand, wird mit einem Hinweis auf den Autor abgeschlossen, welcher die besagten Angaben sammelte und sie im Jahre 1678 als Provinzialminister und Generaldefinitor ordnete „Mi Lector, ora pro eo“.

Als Ergänzung der chronikartigen Eintragungen Sannigs können topographische Bilder der schlesischen Städte gelten, die in der zweiten Hälfte des 18. Jahrhunderts von Friedrich Bernard Werner angefertigt wurden. Trotz gewisser Ungenauigkeiten erlauben sie es, die vom Provinzial der Reformaten angefertigten Beschreibungen miteinander zu vergleichen. Man kann sie grundsätzlich in zwei Gruppen aufteilen. Einerseits sind das die Ansichten von Städten (sowohl die Veduten ${ }^{15}$

15 F. B. Werner, Scenographia urbium Silesiae, (1737-1752), P. 11 (Breslau, Namslau, Leobschütz), 19 (Neiße), 23 (Liegnitz, Goldberg), 35 (Jauer), 39 (Gross Glogau, Troppau, Ratibor), s. http://www.bibliotekacyfrowa.pl/dlibra/ docmetadata?id=15058, (aufgerufen: 12.06.2017); ders., Topographia seu Compen- 
als auch die Stadtplän ${ }^{16}$ ), dank denen die Beobachtung der Klostergrundlegung in der Stadtstruktur möglich ist. Anderseits soll man die Ansichten von einzelnen Reformaten-Klöster unterscheiden, die bei den mehr detaillierten Beschreibungen von konkreten Klosteranlagen hilfreich sein können ${ }^{17}$. Dies kann man als Ausdruck seiner individuellen Vorstellung von den Grenzen des franziskanischen Raums betrachten. Sie sind in Bezug auf das Medium (die Chronik) auch im Gestaltungsprozess des Bewusstseins der ganzen Provinz von wesentlicher Bedeutung.

\section{DIE GRENZEN}

Die Analyse der Grenzen des franziskanischen Raums lenkt die Aufmerksamkeit des Forschers in erster Linie auf die Lage des Gebiets und die Elemente der Bebauung, die diesen Raum bestimmen. Alles betrachtet man in einem breiteren Zusammenhang der lokalen architektonischen und urbanen Struktur. In Anlehnung an die chronikartigen Aufzeichnungen Sannigs sowie die Zeichnungen Werners kann man feststellen, dass alle Reformatenklöster in Schlesien an den Stadträn-

dium Silesiae, pars V, P. 17/25 (Gross Glogau), s. http://www.manuscriptorium.com/ apps/index.php?direct=record\&pid $=U L W$ -rec1314275595_221\#search, (aufgerufen: 12.06.2017); ders. Topographia oder Prodomus delineati Silesiae Principatus Lignicensis [...], P. 168-169 (Goldberg), s. http://www.bibliotekacyfrowa.pl/dlibra/ doccontent?id=14652, (aufgerufen: 12.06.2017).

16 Ders., Topographia seu Compendium Silesiae, pars V, P. 48-49 (Gross Glogau); ders. Topographia oder Prodomus delineati Silesiae Ducatus Wratislaviensis [...], P. 194-195 (Breslau), 582-583 (Namslau).

17 Ders., Topographia seu Compendium Silesiae, pars V, P. 53/65 (Gross Glogau); ders. Silesia in Compendio seu Topographia das ist Praesentatio und Beschreibung des Herzogthums Schlesiens [...] Pars I, P. $337 / 427$ (Neiße); ders. Topographia oder Prodomus delineati Silesiae Ducatus Wratislaviensis [...], P. 279/327 (Breslau), 429/585 (Namslau); ders. Topographia oder Prodomus delineati Silesiae Principatus Lignicensis [...], P. 52/58 (Liegnitz), 135/171 (Goldberg). 
dern gelegen waren, allerdings innerhalb der Stadtmauern, größtenteils in ihrer nächsten Umgebung, nicht weit von den Stadttoren entfernt. Die Ausnahme bildet das Kloster in Neiße, das von Grund auf „extra Portam Telonariam [...] in suburbio“ neu gebaut wurde ${ }^{18}$. In Bezug auf die städtische Topografie wies Sannig möglichst präzise auch auf die Lage des besetzten Grundstücks wie auch auf die charakteristischen Orientierungspunkte hin, darunter größtenteils auf die besagten Tore: in Namslau war es „non procul a porta Wratislaviensi versus Burgum “19, und in Glogau „ad celebre flumen Oderam, et e diametre secundum latus septentrionale respicit Canonicatum "20. Dort, wo es möglich war, bestimmte er den Platz- bzw. den Straßennamen, wo sich ein Kloster befand, sorgte zugleich dafür, das Äquivalent vulgo zu nennen, wie es im Falle der Breslauer „Platea Canina, vulgo Hundegasse“21, der Jauerer "platea S. Adalberti, vulgo die Olbrichtssgasse“22 oder auch der Namslauer „platea Judaica, vulgo Burgplatz" ${ }^{23}$ ebenfalls zu beobachten ist.

Die Beschreibungen der Lage der Klöster der Reformaten entsprechen deren Platzierung auf den Zeichnungen von Friedrich Bernard Werner. Sie enthalten zusätzlich eine Reihe von neuen Informationen, die in den topographischen Beschreibungen von Sannig nicht vorhanden sind. Beispielsweise wird die von Sannig genannte Klosteranlage in Namslau in der Nähe des Fürstenschlosses und ihre Einbindung in die städtischen Befestigungsanlagen bekannt. Dank des Panoramas der Stadt von Werner ist ersichtlich, dass die Ganzheit sich im südwestlichen Teil der Stadt befand, in unmittelbarer Nähe des Flusses und der niedrigen städtischen Bebauung. Ein besonderer Vorteil dieser Visua-

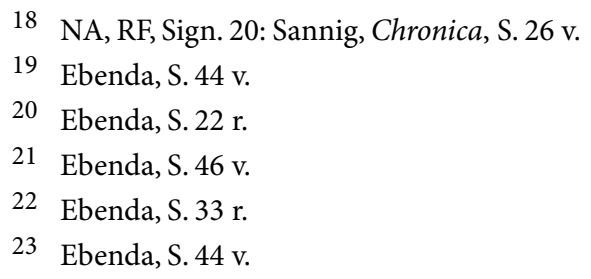


lisierungen bleibt jedoch die Möglichkeit, die Gebäude der Reformaten im urbanistischen Raum präziser zu erfassen, also in jenem Zusammenhang, der durch die damalige Struktur der städtischen Bebauung gestaltet wurde. Dieser Kontext lässt die Größe der angesprochenen Klosteranlagen im Vergleich mit den umliegenden Vierteln annäherungsweise erfassen und darin auch eine Art Dominanten bemerken, die den städtischen Raum mitkomponierten. Als ein gesonderter Aspekt bleiben die Angaben, die den detaillierteren Zeichnungen von einzelnen Kloster- und Kirchengebäuden zu entnehmen sind. Werner hat deren architektonische Details und spezifische Blockplanung aufgezeigt und dazu beigetragen, diese konkreten Gebäude im städtischen Raum auszuzeichnen. Es kann also von einer starken Korrelation und gegenseitiger Ergänzung von Stadtaussichten und -plänen und ausgewählten detaillierten Zeichnungen die Rede sein. Die letzten wurden von Werner als relevant eben in Hinblick auf die städtische Struktur gezeichnet. Er sah darin auszuzeichnende Orientierungspunkte. Auch in dieser Hinsicht sind die Arbeiten von Werner und Sannig ähnlich. Es scheint, dass eine so klare Anknüpfung Sannigs an die zweisprachigen Stadtnamen der Orientierungspunkte nicht nur der genauen räumlichen Identifikation eines Grundstücks diente, sondern auch betonen sollte, dass etwas zu der Stadtstruktur gehörte. Das Reformatenkloster ist daher ein klarer Bestandteil des städtischen Raums wie das Breslauer Tor oder das Schloss. Somit wird auf eine gewisse Beständigkeit hingewiesen.

Wie ich es oben schon bemerkt habe: Die Reformaten kommen in die schlesischen Städte nicht, sondern sie kehren in ihrem Selbstverständnis zurück. Ihr Dienst in der Stadt des 17. Jahrhunderts gilt also als Fortsetzung davon, was die Reformation im 16. Jahrhundert unterbrochen hat. Ferner sind die Reformaten auch die direkten Nachfolger der Observanten. Dies geht darauf zurück, dass bisher die ganze böhmische Observantenprovinz 1660 die reformierten Statuten und die gleiche Verfassung annahm, was zu ihrer Umgestaltung in eine neue St.-Wenzel-Reformatenprovinz führte. In Anlehnung an die 
nachtridentinische Rhetorik war die Aussage klar: Die Reformaten sind die wiedergeborenen und im Geiste der Rückkehr zum ursprünglichen Franziskanismus reformierten Observanten. Die alten Mauern erhielten also eine neue Qualität. In der Chronik Sannigs wird dies zusätzlich hervorgehoben, und zwar in solchen Wendungen wie: „Patres Observantes, qui hodie Reformati appellantes" 24 . Eine wesentliche Rolle spielt in diesem Prozess die Tatsache, dass alle Klöster außer Breslau und Neiße, wo Kirchen und Klöster an ganz neuen Stellen gebaut wurden, als spätmittelalterliche Bauten der Observanten aus dem 15. Jahrhundert galten. Vor diesem Hintergrund zeichnete sich nur das Kloster in Namslau ab, das im 14. Jahrhundert für die Minoriten gestiftet worden war. Die Lage der Klöster und Kirchen in der nächsten Umgebung der Stadtbefestigungsanlagen erinnert an die Idee, die geistlichen Bauwerke als geistige Stadtbastionen zu betrachten, welche dem System der Festigungswerke ähnelten ${ }^{25}$. Und höchstwahrscheinlich wurde die Hervorhebung Sannigs in Bezug auf eine solche Lage so verstanden. In Wirklichkeit ging die Wahl der Lage vor allem auf die Entscheidung der Stifter zurück, die einen Teil ihres Grundstücks, darunter, wie im Falle von Namslau, des herzoglichen Jurisdiktionsbezirkes, für den Bau bestimmten. Das vom Orden benutzte Grundstück befand sich also in der Stadt, blieb mit der Stadt verbunden, war aber hinsichtlich des Eigentums von der Stadt unabhängig ${ }^{26}$.

Die architektonische Form spielte auch eine wesentliche Rolle in der Komposition des städtischen Raumes. In dieser Hinsicht kann die Architektur der Gebäude schlesischer Reformaten in zwei Gruppen aufgeteilt werden: Die Gebäude, die an der Wende des 17. zum 18.

24 Ebenda, passim.

25 Klima, Przestrzeń, S. 38; A. Witkowska, Przestrzeń sakralna późnośredniowiecznego Krakowa, in: Ecclesia et civitas. Kościół i życie religijne w mieście średniowiecznym, hg. v. H. Manikowska, H. Zaremska, (2002), S. 37.

26 G. Wąs, Klasztory franciszkańskie w miastach śląskich i górnołużyckich XIII-XVI wieku, (Acta Universitatis Wratislaviensis 2222. Historia 142, 2000). 
Jahrhundert neu erbaut wurden (häufig an ganz neuen Orten) ${ }^{27}$, und diejenige, die als Observanten-Kirchen- und Klostergebäude aus der zweiten Hälfte des 15. Jahrhunderts aufgrund der Rückkehr der Franziskaner nach dem dreißigjährigen Krieg mehr oder weniger umgebaut (barockisiert) wurden ${ }^{28}$.

Außerdem ist es auch möglich, chronologisch und stilistisch einige Etappen zu unterscheiden, in denen die barocke Architektur der Reformaten sich gestaltete - unter Berücksichtigung beider oben genannter Gruppen. Der erste Zeitraum würde beginnen mit der Wiedererlangung des ersten Gebäudes in Glogau in der ersten Dekade des 17. Jahrhunderts, danach folgten die nächsten: in Neisse, Jauer, die mehr oder weniger unter Verwendung der frühbarocken Dekorationsmotive revitalisiert wurden. Der zweite Zeitraum zeichnet sich aus durch hohe Bauaktivität und fällt in die 80er Jahre des 17. bis Anfang des nächsten Jahrhunderts. Damals wurden die Klosteranlagen in Breslau, Ratibor oder Namslau realisiert, in denen eine gewisse Festigung der frühbarocken Strömungen und deren einigermaßen geschickte Verwendung sichtbar wird, häufig unter Mithilfe von und nach italienischen Meistern. Der dritte Zeitraum bildet eine späte Etappe der schlesischen Reformaten-Architektur und erstreckt sich, mit unterschiedlicher Intensität, auf die ganze erste Hälfte des 18. Jahrhunderts und umfasst somit die Klosteranlagen in Liegnitz und Goldberg bis hin zum Umbau des Kirchen- und Klostergebäudes in Leobschütz. Diese Bauten, insbesondere die kirchlichen, sind gekennzeichnet durch den Wandel des Spätbarocks und des Frührokokos - im Bereich der eingesetzten räumlichen Lösungen und des konsequenten Verzichts auf die für vorige Zeiträume so charakteristische statische Tektonik der Blöcke, zu Gunsten deren Ondulation, der künstlerischen Umformungen und der plastischen Modellierung.

27 Eine solche Situation fand in Neisse, Breslau, Ratibor, Liegnitz und Leobschütz statt.

28 Die Klöster in: Namslau, Goldberg, Troppau, Glogau und Jauer. 
Die Situation der Restitutionen der alten Franziskanerhäuser wurde daher zum wichtigen determinierenden Faktor, der sowohl über die Lage der spätneuzeitlichen Reformatenklöster in den schlesischen Städten als auch größtenteils über ihr Aussehen entschied. Sie zeichneten sich schließlich durch die Exposition der gotischen Elemente aus, die man einer präzisen Barockisierung im Geiste der gegenreformatorischen Stilistik unterzog. Nur in Neiße, Breslau und Leobschütz erfüllte man völlig die Voraussetzungen der neuen Architektur, die an das Vorbild von Il Gesù (Jesuskirche) in Rom anknüpfte. Der Kirchenbau mit einer für das Barockzeitalter klassischen Fassade mit einem Giebel mit Volutenfluss galt als eine im protestantischen Raum eindeutig erkennbare Aussage und Zeichen der Anwesenheit der Vertreter der römisch-katholischen Kirche in einer neuen nachtridentinischen Form.

In seinen eher skizzenhaften Beschreibungen des Raums berücksichtigt Sannig vor allem die Pläne und die Formen der einzelnen Objekte sowie ihre Lage auf einem Grundstück und gegenüber der lokalen Bebauung. Eine vollständige räumliche Orientierungshilfe bieten die erwähnten Größen aller Objekte, die Sannig in drei metrischen Systemen aufzeichnete: klassische Maßeinheiten wie Fuß und Elle, die gleichen in der böhmischen und Neißer Variante sowie in den Beschreibungen der Häuser in Jauer und Troppau eine Einheit orgia. Somit kann man feststellen, dass die durchschnittliche Größe eines Grundstücks, das von den Brüdern besetzt war und auf dem sich eine Kirche, ein Kloster, ein großer Garten und im Falle von Namslau und Jauer ein Friedhof befanden, $5500 \mathrm{~m}^{2}$ betrug. Eine größere Abweichung findet sich beim Glogauer Grundstück mit über $8000 \mathrm{~m}^{2}$ und beim Neißer mit knapp $2800 \mathrm{~m}^{2}$. Ein vorherrschendes Element des franziskanischen Raums, und zwar hinsichtlich der Größe, war der Klostergarten, dessen längere Seite sogar $70 \mathrm{~m}$ betragen konnte. Gemäß den Vorschriften der Ordensregel waren die Klöster und die Kirchen der Reformaten nicht groß und am häufigsten an einer Gebäudeseite miteinander verbunden. 
Die Grenzen des franziskanischen Sakralraums sollte man jedoch breiter als die wirklichen Grundstücksgrenzen wahrnehmen. Im Falle der schlesischen Städte könnte man Trevor Johnson zufolge von konfessionellen Grenzen sprechen, die ich als ein kulturelles, durch materiellen Raum bestimmtes Gebiet, auf dem die konfessionellen Identitäten bestimmt werden, verstehe ${ }^{29}$. Es geht also um unsichtbare Grenzen, die sowohl Ritual, Brauch als auch Geschichte oder Gedächtnis (also alles, was der Raum versinnbildlicht) bilden. Diese „mentalen Grenzen“ halte ich für ein Konstrukt, das in gewissem Sinne einen Kontext bildet. Denn es reicht nicht aus, solche Grenzen nur zu setzen. Man sollte sie auch verstehen. Erst dann ist es deutlich, welchen Raum diese sowohl wirklichen als auch mentalen Grenzen bestimmen.

\section{Die BEDEUTUNG}

Die Bedeutung des franziskanischen Raums in den schlesischen Städten des 17. Jahrhunderts möchte ich in Anlehnung an die These Paul Ricoeurs untersuchen, laut dem die Stadt sowohl gesehen als auch gelesen wird ${ }^{30}$. Die Erzählung und die Gestaltung machen nämlich vergleichbare Eintragungen: die eine im Bereich des Bestehens, die andere der Beständigkeit des Materials. Jeder neue Bau gehört zum städtischen Raum ähnlich wie eine Erzählung zum Netz der Intertextualitä ${ }^{31}$. Deswegen kann man aus der Sicht der Bedeutungen, die in der Architektur und im Raum verschlüsselt sind, die Stadt als eine Form des Kulturtextes betrachten, der über die Grenzen der Historizität oder der Ästhetik hinausgeht und zum Träger der überzeitlichen, die

29 T. Johnson, Defining the confessional frontier: Bavaria, the Upper Palatinate and counter-reformation 'Historia Sacra', in: Frontiers and the writing of history. 1500-1800, hg. v. S. G. Ellis, R. Eßer, (2006), S. 151.

30 P. Ricoeur, Pamięć, historia, zapomnienie, (2012), S. 199.

31 Ebenda, S. 198. 
kollektive Identität und das kulturelle Gedächtnis bestätigenden Werte wird $^{32}$.

Die räumliche Metaerzählung der Stadt besteht aus vielen den Orten, Objekten oder Räumen zugeschriebenen Einzelerzählungen, d. i. kleineren Einheiten. Dazu gehören auch die Räume, die als Sakralräume identifiziert werden. Die Exposition der kommunikativen Funktion einer Stadt und ihrer einzelnen Elemente - der Sphären, Räume, der Bebauung - sollte es ermöglichen, die Inhalte, derer Träger sie sind, abzulesen und zu interpretieren. Diese Analyse bestimmt sehr genau die diesen Raum beschreibende Geschichtsschreibung. Sie ist zugleich sowohl ein gewisser Führer durch den besagten Raum als auch ein ihn bestimmender Kommentar.

Darüber hinaus ist der Sakralraum eine besondere Grundlage für das Zusammentreffen mit der Vergangenheit und die Konfrontation mit kollektiven Werten, die im Sakralisierungsprozess, auch im Sinne im Raum verschlüsselter Gedächtnisstrukturen, gegeben werden ${ }^{33}$. Damit sind hingegen die Identitätsbildungsprozesse verbunden, welche man als eine Selbstidentifikation mit den Werten, die in diesem Raum repräsentiert sind, begreifen kann. Die wesentliche Rolle der Geschichte und des Gedächtnisses sowohl in der Sakralisierung des Raums als auch in der Identitätsbildung geht daraus hervor, dass "die Geschichtsschreibung zeitliche und räumliche Komponenten hat. Sie ist nicht nur eine Erzählung der Zeit, sondern auch des Raumes; sie kombiniert chronologische mit geografischen oder topografischen Aspekten " ${ }^{34}$. Man kann also sagen, dass sich jede Geschichte in einem bestimmten Raum vollzieht. Die Zeit und der Raum sind aber zwei

32 A. Assmann, Między historia a pamięcią. Antologia, hg. v. M. Saryusz-Wolska, (2013), S. 30-31.

33 Kowalewski, Kowalewska, Katedra, S. 78.

34 R. Esser, S. Rau, M. Stercken, Raum, in: Geschichte schreiben. Ein Quellen- und Studienhandbuch zur Historiografie (ca. 1350-1750), hg. v. S. Rau, B. Studt, (2010), S. 445 . 
Vektoren, unter deren Einfluss die Erzählung, die als Grundlage der Identitätsbildung gilt, entsteht.

In dieser Hinsicht gestaltet sie den Raum beständig und verständlich mit, indem sie auf das Bewusstsein und das Gedächtnis des Empfängers zurückgeht. Es ist sehr sichtbar auch in der Beziehung zwischen der Chronik Bernard Sannigs und den faktischen franziskanischen Räumen in den schlesischen Städten. Sannig, der die bestimmten Orte mit historischen Ereignissen zu verbinden sucht und zugleich auf ihre Bedeutung für den zeitgenössischen Reformatenkonvent hinweist, bildet eine Art Topografie des franziskanischen Gedächtnisses. Diese Erscheinung kann man beispielsweise anhand von Breslau untersuchen. Wie ich es bereits erwähnt habe, errichtete man das St.-AntoniusKloster am Ausgang des 17. Jahrhunderts an einer völlig neuen Stelle ${ }^{35}$, interessanterweise auch in der Nähe der Stadtmauern. Das Grundstück wurde dem Orden vom Breslauer Stadtrat für den Bau nach über 100 Jahren zuerkannt, welche er um die Wiedererlangung des 1522 beschlagnahmten Klosters und der St.-Bernardus-Kirche aus der Mitte des 15. Jahrhunderts, die auf Anregung des sich in Breslau aufhaltenden St. Johannes Kapistranus entstanden war, hindurch kämpfte. Das alte Haus der Observanten war also nicht zurückzugewinnen. Die Brüder kehrten in die Stadt zurück, aber in eine völlig andere Umgebung. Man kann den Eindruck gewinnen, dass die erweiterte Beschreibung der Geschichte der Breslauer Observanten und später auch der Kämpfe um die Restitution, an der Sannig selbst höchst persönlich beteiligt war, neben der eindeutigen Hervorhebung der Rechte der Reformaten auf den Dienst in Breslau auch der Herausbildung einer neuen, sich auf das historische Bewusstsein stützenden Identität des lokalen Konvents und seiner erneuten Verbindung mit der Stadt diente. Die Achsen der sich herausbildenden Identität waren drei Ereignisse: der Besuch St. Johannes Kapistranus' in der Stadt und die damit verbundene Initiative

35 M. Musiał, Okoliczności powrotu franciszkanów reformatów do XVII-wiecznego Wrocławia w świetle Instrumentum Transactionis, „Studia Śląskie“, 79 (2016), S. 35-56. 
des St.-Bernardus-Klosterbaus 1453, die Verbannung der Observanten 1522 sowie die Rückkehr der Reformaten 1678. Wenn Sannig diese Beschreibungen auf den Raum zurückführt, erwähnt er zweimal eines der Stadttore, und zwar das Nikolai Tor. Es lag im Westteil der Stadt und wurde zum ersten Mal in Bezug auf die Beschreibung der Verbannung der Observanten aus Breslau im Juni 1522 erwähnt. Laut dem Chronisten führte die Prozession der Ordensbrüder von ihrer St.-Bernardus-Kirche bis zum Nicolai Tor, „durch das sie einst mit Kapistranus eingeführt worden waren“. Zum zweiten Mal wurde das Nicolai Tor in der Beschreibung eines neuen Grundstücks an der St.Antonius-Straße, früher Hundegasse, erwähnt. Das Grundstück erhielt der Orden als Wiedergutmachung und musste dafür alle Rechte auf die St.-Bernardus-Einrichtung aufgeben. Sannig, der zugleich seine Länge und Breite anführte, wies darauf hin, dass es innerhalb der Stadtmauern gelegen ist, nicht weit vom Nicolai Tor, durch das früher unsere verbannten Brüder Observanten die Stadt verlassen mussten ${ }^{36}$. Im Resultat kann man vom Gedankensprung sprechen, der sich in der Beschreibung des Tores auf die Erinnerung an ein chronologisch folgendes Ereignis bezog. In der Beschreibung des Grundstücks knüpft der Chronist an keinen Eingang Kapistranus' in die Stadt mehr an, sondern an die Verbannung seiner Nachfolger 1522. Diese Erzählung wurde später im Prozess der historischen Symbolisierung Kapistranus' anscheinend gekürzt. Sein Bildnis spiegelt also im Breslauer Konvent nicht nur das Gedächtnis für den heiligen Bekenner, Bezwinger der Türken und den eifrigen Anhänger der Observanz wider, sondern auch das verlorene St.-Bernardus-Kloster, dessen Begründer er war. Neben dem Hinweis auf die Einrichtung selbst erinnert es auch an die wichtigen Ereignisse, d. i. an die Stiftung und die Verbannung. Kapistranus wurde daher zum Vermittler zwischen diesen beiden Stufen der Observantengeschichte in der Stadt. Man sollte sich auch nicht wundern,

36 NA Praha, RF, Sign. 20: Sannig, Chronica, S. 46 v. 
dass einer der Seitenaltäre in der neuen Reformatenkirche in Breslau eben ihm gewidmet ist.

Die Erzählung über eine ähnliche Wanderung durch die Stadt auf den Franziskanerspuren wiederholte Sannig auch in Neiße, das ihm besonders lieb war, wo die Observanten zweimal die Lage wechselten und anschließend ein neues Kloster in der Vorstadt gründeten. Sannig betonte, dass einer der Altäre Maria Magdalena gewidmet wurde. Das gleiche Prädikat hatte das ehemalige Kloster am Brüder-Thor. Es gab dort auch eine wundervolle Figur von der Gottesmutter Maria, die die Brüder aus dem früheren Kloster mit sich brachten. Alle diese Anknüpfungen an die Vergangenheit weisen eindeutig auf die Beständigkeit des Dienstes hin, zeigen den Reformaten, den Zeitgenossen Sannigs, den Sinn und bestätigen den Missionsdienst unter den Protestanten, der durch das Fortbestehen bestimmt wird.

Die oben skizzierte Gestalt Johannes Kapistranus ${ }^{37}$ führt das letzte der Elemente ein, das ich in Bezug auf die zu bestimmende Bedeutung des franziskanischen Raums in der Stadt hervorheben möchte. Es geht nämlich um die Ikonographie. Man kann von einer engen Beziehung zwischen den stilistischen Formen und Gestaltungen der Reformatenbauten und den Bildprogrammen ihrer Innenräume sprechen. Hervorzuheben ist dabei eine gewisse schlesische Spezifik dieser Bebauung: Ein wenig wider die scharfe, die Armut empfehlende Gesetzgebung der Reformaten wurden ihre Kirchen in den schlesischen Städten von den hervorragendsten Künstlern des schlesischen Barockzeitalters errichtet und ausgestattet: Matthias Steinl und Michael Willmann. Sie zeichneten sich also durch die Meisterschaft ihrer Ausführung und der benutzten Formen aus, die man aber vorsätzlich an die potentialen Evangelisierungsbedürfnisse anpasste, indem man die Jesuiten oder Zisterzienser nachahmte. Die Kirche wurde maßvoll geziert, man berücksichtigte

37 M. Elbel, Kult sv. Jana Kapistrána v českých zemích, „Historia artium. Acta Universitatis Palackianae Olomucensis, Facultas philosophica. Philosophica-Aesthetica Univerzita Palackého Olomouc“, 1993, 2 (1998). 
aber die Wirkkraft der Bildnisse. In Bezug auf die Architektur spiegelte das Kloster tatsächlich die Idee eines armseligen Lebens der Brüder wider. Einfache und raue Fassaden, Bauten mit einem oder zwei Stockwerken auf dem Grundriss eines Rechtecks, mit gewöhnlichem Innenhof, alles der Regel der Nützlichkeit untergeordnet ${ }^{38}$. Alle Reformaten-Bauten sollten ein sichtbares Armutszeichen sein, „[...] ne tante pauperati a nobis promissae, quod patet oculis, contrarium foris

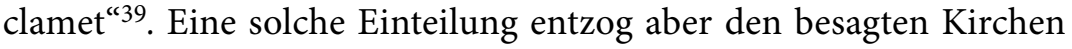
keinen franziskanischen Geist. Von der Beziehung zum Orden zeugten eindeutig die Patrozinien sowie die Heiligenbildnisse, die für die Reformaten und die nachtridentinische Kirche ${ }^{40}$ charakteristisch waren. Unter den Patronen der Franziskaner, die von Sannig mehrmals erwähnt werden, befinden sich vor allem St. Franziskus von Assisi und St. Antonius von Padua, die für die ganze Franziskanerfamilie gemeinsam sind. Weiter wollten die Brüder ihre heiligen Mitbrüder, die um die Reform bemüht waren, besonders zur Schau stellen: St. Johann Kapistranus oder Peter von Alkantara. Das ganze Bild ergänzten die Vorstellungen von den lokalen Heiligen: Wenzel, Hedwig von Schlesien oder Anna

38 M. Musiał, Użyteczność w procesie kształtowania formy architektonicznej - sugestia czy imperatyw? Na przykładzie poreformackiego kościoła i klasztoru św. Antoniego we Wrocławiu, in: Ars et scientia. I Łódzkie Spotkanie Studentów Historii Sztuki (Łódź 11-12.01.2013), hg. v. K. Stefański, (2014), S. 121-146.

39 Statuta Provincialia Almae Provinciae Boemiae S. Wenceslai Ordinis Minorum Sancti Patris Francisci Reformatorum In Capitulo provinciali Novae Domiad S. Catharinam Anno 1675 die 12. Mensis Maij celebrato facta [...], (1676); Statuta Provincialia Almae Provinciae Bohemiae S. Wenceslai D. et M. Ordinis minorum sancti Patris Francisci Reform. In pluribus capitulis provincialibus et diffinitoriis legitime condita, ac a duobus ministris Generalibus, F. Francisco Maria de Cremona, et F. Petro Matino Sormanno de Mediolano rite approbata ad iteratam instantiam F. Bernardi Sannig, lectoruis jubilati, totius ordinis patris, ex-commissatii Generalis cismontani, et iterati ministri provincialis ejusdem provinciae, (1686), S. 37.

40 R. Po-chia Hsia, Gegenreformation. Die Welt der katholischen Erneuerung 1540-1770, (1998), S. 165-207; S. Samerski, „Wie im Himmel, so auf Erden“? Selig- und Heiligsprechung in der Katholischen Kirche 1740 bis 1870, (2002). 
von Böhmen ${ }^{41}$. Die Programme zeichneten sich darüber hinaus durch die für die Franziskanerspiritualität typische Vorliebe für die Passion und die Gottesmutter Maria aus. Es scheint, dass eben diese für den franziskanischen Raum distinktiven Elemente als perzeptive Grenzen, d. i. Grenzen zweiten Ranges gelten können. Sie ergänzen hauptsächlich die faktischen Grenzen, die von den Wänden der Gebäude und Mauern der Gärten bestimmt wurden. Sie sind es, welche im Zusammenhang mit der Geschichtsschreibung, die den Orten und Räumen große Bedeutung zumisst, eine verschlüsselnde Struktur bilden.

Die frühneuzeitliche Historiographie der böhmischen Reformaten in Schlesien entwickelte sich auf besondere Art und Weise um den Begriff „Rückkehr“. Er wurde nämlich als ein gewisses „Schlüsselwort" in der inneren provinziellen historiographischen Narration (P. Bernard Sannig) genutzt. Der Begriff wurde oft mit einem anderen reformationsbezogenen Begriff verbunden: „Vertreibung“. Diese beiden Begriffe bildeten einen gewissen Kern der neuzeitlichen Konstruktion der Vergangenheitsdarstellung, der in Verbindung mit weiteren Begriffen, wie die der "Vertreibung" vorangegangenen „Stiftung", zu einem komplexen geschichtlich-juristischen Argument für die „Rückkehr" der Reformaten in die Städte Schlesiens geworden war. Darin kommt auch die Unterscheidungskraft Schlesiens im Vergleich mit der gesamten böhmischen Provinz der Reformaten zum Ausdruck: Alle Observanten-Kloster wurden in der Reformationszeit verlassen, schon im 17.-18. Jahrhundert kamen die Reformaten zurück. In diesem Zeitraum kommen keine neuen Reformaten-Stiftungen in Schlesien vor - alle wurden wiedererlangt und zurückgegeben, sowohl dem gesellschaftlichen Bewusstsein als auch dem städtischen Raum.

Die beschriebene Geschichtskonstruktion und die Rückkehr der Franziskaner im 17. Jahrhundert, die auf den Blättern der Chronik

41 M. Elbel, On the side of the angels: Franciscan communication strategies in early modern Bohemia, in: Religious Exchange in Europe, 1400-1700, hg. v. H. Schilling, I. Tóth, (2006), S. 338-359. 
von Sannig häufig als reintroductio oder renovatio bezeichnet wurde, entspricht gut dem posttridentinischen Programm der Erneuerung der katholischen Kirche im Geiste der kontrareformatorischen Rhetorik. Auf einer weiteren Ebene fanden die Ideen unter anderen in den Begriffen Ecclesia triumphans (triumphierende Kirche = die nach den Reformen des Konzils triumphierend zurückkehrte) ihren Ausdruck. Die schlesische Besonderheit des Ordens hat zusätzlich die Konzepte der Erneuerung im Geiste der strengen Auslegung der Franziskusregel gestärkt und zu diesem Zweck die Idee der radikalen Rückkehr zu den Quellen des seraphischen Lebens. Nicht ohne Bedeutung bleibt beispielsweise der Name der Observanten-Gemeinschaft selbst - die Reformaten.

In diesem historiographisch-topographischen Prozess kommt Sannig sowohl als Begründer als auch gewissermaßen als Führer vor. Das systematisierte Beschreibungsformular jedes der Franziskanerkonvente der Provinz führt den Leser in den der Chronologie eindeutig untergeordneten franziskanischen Stadtraum ein. Interessanterweise sind nicht alle Orte, die für die Reformaten wichtig waren, eng mit der Umgebung des Klosters verbunden, wovon beispielsweise das Breslauer Nicolai Tor zeugt. Die Verschlüsselung des Raums, und zwar in Bezug auf die am Anfang dargestellten Schlussfolgerungen, die Tätigkeit zugunsten der Konsolidierung und Herausbildung des Identitätskonstrukts erlaubt es Sannig, gewissermaßen mit der Zeit zu spielen. Bei der Erinnerung an die einzelnen Schichten knüpft er an die früheren Ereignisse an, erinnert an die seit langem abwesenden Personen, und zwar mit dem Ziel, dadurch eine gewisse Gerechtigkeit in der Geschichte zu schildern, laut der die Reformaten, indem sie den Willen Gottes erfüllen, zu den protestantischen Städten Schlesiens zurückkehren und den Ruhm der wiedergeborenen Kirche in die Welt tragen.

Übersetzt von Liliana Lewandowska, teils korrigiert von Joanna Mielczarek 


\section{GRANICE I ZNACZENIE PRZESTRZENI SAKRALNEJ FRANCISZKANÓW REFORMATÓW W NOWOŻYTNEJ PRZESTRZENI MIEJSKIEJ NA ŚLĄSKU}

(STRESZCZENIE)

Po okresie reformacji, w wyniku której przestały funkcjonować niemal wszystkie klasztory czeskiej prowincji, franciszkanie reformaci stopniowo powracali do miast śląskich (w XVII wieku). Odradzanie się tej prowincji polegało nie tylko na odzyskiwaniu dawnych klasztorów, lecz wiązało się także z koniecznością kreacji nowej tożsamości wspólnoty. Istotne znaczenie miała przy tym pamięć o wcześniejszych dziejach zakonu na tych terenach, także o posiadanych dobrach. W artykule podjęto temat mentalnej „przestrzeni“ franciszkańskiej na Śląsku w XVII wieku, którą w świadomości członków tego zakonu tworzyły niegdysiejsze oraz aktualnie posiadane klasztory.

\section{GRENZEN UND BEDEUTUNG DES SAKRALRAUMS DER FRANZISKANER-REFORMATEN IM FRÜHNEUZEITLICHEN STADTRAUM IN SCHLESIEN}

\section{(ZUSAMMENFASSUNG)}

Nach der Reformation, in deren Folge fast alle Klöster der böhmischen Provinz nicht mehr bestanden, kehrten die Franziskaner-Reformaten allmählich in die schlesischen Städte zurück (17. Jahrhundert). Die Wiederbelebung dieser Provinz erfolgte nicht nur mit der Wiedererlangung der ehemaligen Klöster, sondern war auch mit der Notwendigkeit der Schaffung einer neuen Gemeinschaftsidentität verbunden. Eine relevante Bedeutung hatte dabei die Erinnerung an die frühere Geschichte des Ordens in diesem Gebiet und an ihre Güter. Im Beitrag wurde das Thema des mentalen „Franziskaner-Raumes" in Schlesien im 17. Jahrhundert thematisiert, das im Bewusstsein der Mitglieder des Ordens durch ehemalige und aktuelle Klöster virulent war.

Übersetzt von Renata Skowrońska 


\section{BORDERS AND SIGNIFICANCE OF THE SACRED SPACE OF FRANCISCAN REFORMERS IN THE EARLY MODERN CITY SPACE IN SILESIA}

(SUMMARY)

After the Reformation, during which almost all monasteries in the Czech province ceased to operate, Franciscan reformers were gradually returning to the Silesian towns (in the $17^{\text {th }}$ century). The rebirth of the province consisted not only in regaining former monasteries, but was also connected with the necessity to create a new identity of the community. What played an important role here was the memory about the history of the monastery in this area and their estates. The article addresses the issue of the mental Franciscan "space" in Silesia in the $17^{\text {th }}$ century, which consisted of former and present monasteries owned by the members of the Franciscan community.

Translated by

Agnieszka Chabros

\section{Słowa kluczowe / Schlagworte / Keywords}

- franciszkanie reformaci; Śląsk; przestrzeń sakralna

- Franziskaner-Reformaten; Schlesien; Sakralraum

- Franciscan reformers; Silesia; sacral space

\section{BIBLIOGRAFIA / BIBLIOGRAFIE / BIBLIOGRAPHY}

\section{ŹRÓDŁA ARCHIWALNE / ARCHIVALISCHE QUELLEN / ARCHIVAL SOURCES}

Národní Archív Praha, Archiv České fratiškánské Provincié, Sign. 20: B. Sannig, Chronica de origine et constitutione Provinciae Bohemiae Ordinis Fratrum Minorum S. Francisci Reformatorum,(1678). 


\section{ŹRÓDłA DRUKOWANE / GEDRUCKTE QUELLEN / PRINTED SOURCES}

Statuta Provincialia Almae Provinciae Boemiae S. Wenceslai Ordinis Minorum Sancti Patris Francisci Reformatorum In Capitulo provinciali Novae Domiad S. Catharinam Anno 1675 die 12. Mensis Maij celebrato facta [...], (1676).

Statuta Provincialia Almae Provinciae Bohemiae S. Wenceslai D. et M. Ordinis minorum sancti Patris Francisci Reform. In pluribus capitulis provincialibus et diffinitoriis legitime condita, ac a duobus ministris Generalibus, F. Francisco Maria de Cremona, et F. Petro Matino Sormanno de Mediolano rite approbata ad iteratam instantiam F. Bernardi Sannig, lectoruis jubilati, totius ordinis patris, ex-commissatii Generalis cismontani, et iterati ministri provincialis ejusdem provinciae, (1686).

Wrbczansky S., Nucleus Minoriticus, seu vera et sincera relatio originis et progressus provinciae Bohemiae, conventuum et residentiarum fratrum et sororum sancti-monialium ordin. minor. S. P. Francisci Strict. Observ. Reform. in Provincia, sub patrocinio Sancti Wenceslai ducis et martyris, Per Bohemiam, Moraviam et Silesiam existentium. Nec non fundatorum, capitulorum, commisariorum, provincialiorum aliorumque memoria dignorum, ex archivis, proto-collis et manuscriptis, sedule collectorum compilatum,(1746).

\section{LITERATURA / LITERATUR / LITERATURE}

Assmann A., Między historia a pamięcią. Antologia, hg. v. M. Saryusz-Wolska, (2013).

Czechowicz J. J., Architektura sakralna Krakowa - sztuka formowania przestrzeni, in: Sztuka tworzenia miejsc, hg. v. S. Hryń, (2008), S. 25-36.

Elbel M., Kult sv. Jana Kapistrána v českých zemích, „Historia artium. Acta Universitatis Palackianae Olomucensis, Facultas philosophica. Philosophica-Aesthetica Univerzita Palackého Olomouc", 1993, 2 (1998).

Elbel M., On the side of the angels: Franciscan communication strategies in early modern Bohemia, in: Religious Exchange in Europe, 1400-1700, hg. v. H. Schilling, I. Tóth, (2006), S. 338-359.

Esser R., Rau S., Stercken M., Raum, in: Geschichte schreiben. Ein Quellen- und Studienhandbuch zur Historiografie (ca. 1350-1750), hg. v. S. Rau, B. Studt, (2010), S. 445-448.

Gapski H., Klasztory zakonów franciszkańskich w Europie Środkowo-Wschodniej w XVIII wieku. Studium przestrzeni franciszkańskiej, (2013).

Jałowiecki B., Czytanie przestrzeni,(2012). 
Jałowiecki B., Społeczne wytwarzanie przestrzeni,(2010).

Johnson T., Defining the confessional frontier: Bavaria, the Upper Palatinate and counter-reformation 'Historia Sacra', in: Frontiers and the writing of history. 1500-1800, hg. v. S. G. Ellis and R. Eßer, (2006), S. 151.

Klima E., Przestrzeń religijna miasta, (2011).

Kowalewski M., Kowalewska D., Katedra jako stereotyp przestrzenny. Przypadek kościoła pw. św. Jakuba w Szczecinie, in: Miasto i sacrum, hg. v. M. Kowalewski, A. M. Królikowska, (2011).

Minař́k K., Provinciál P. Bernard Sannig, učenec, spisovatel a organisátor františkánské provincie (1637-1704), „Časopis pro Katolického Duchovenstwa", 61 (1920)-71 (1930) (publiziert in Teilen).

Musiał M., Okoliczności powrotu franciszkanów reformatów do XVII-wiecznego Wrocławia $w$ świetle Instrumentum Transactionis, „Studia Śląskie“, 79 (2016), S. 35-56.

Musiał M., Użyteczność w procesie kształtowania formy architektonicznej - sugestia czy imperatyw? Na przykładzie poreformackiego kościoła i klasztoru św. Antoniego we Wrocławiu, in: Ars et scientia. I Łódzkie Spotkanie Studentów Historii Sztuki (Łódź 11-12.01.2013), hg. v. K. Stefański, (2014), S. 121-146.

Niezabitowska E., Ewolucja konceptu przestrzeni w teorii architektury, (2008).

Po-chia Hsia R., Gegenreformation. Die Welt der katholischen Erneuerung 1540-1770, (1998), S. 165-207.

Reisch Ch., Die Franziskaner im heutigen Schlesien von Anfange des 17 Jahrhunderts bis zur Säkularisation, ,Zeitschrift des Vereins für Geschichte Schlesiens“, 47 (1913), S. 276-300.

Ricoeur P., Pamięć, historia, zapomnienie, (2012).

Samerski S., „Wie im Himmel, so auf Erden“? Selig- und Heiligsprechung in der Katholischen Kirche 1740 bis 1870, (2002).

Teichmann L., Ordensprovinzen der Franziskaner in Schlesien, „Archiv für Schlesische Kirchengeschichte", 43 (1985), S. 263-274.

Wąs G., Klasztory franciszkańskie w miastach śląskich i górnołużyckich XIII-XVI wieku, (Acta Universitatis Wratislaviensis 2222. Historia 142, 2000).

Witkowska A., Przestrzeń sakralna późnośredniowiecznego Krakowa, in: Ecclesia et civitas. Kościół i życie religijne w mieście średniowiecznym, hg. v. H. Manikowska, H. Zaremska, (2002), S. 37. 


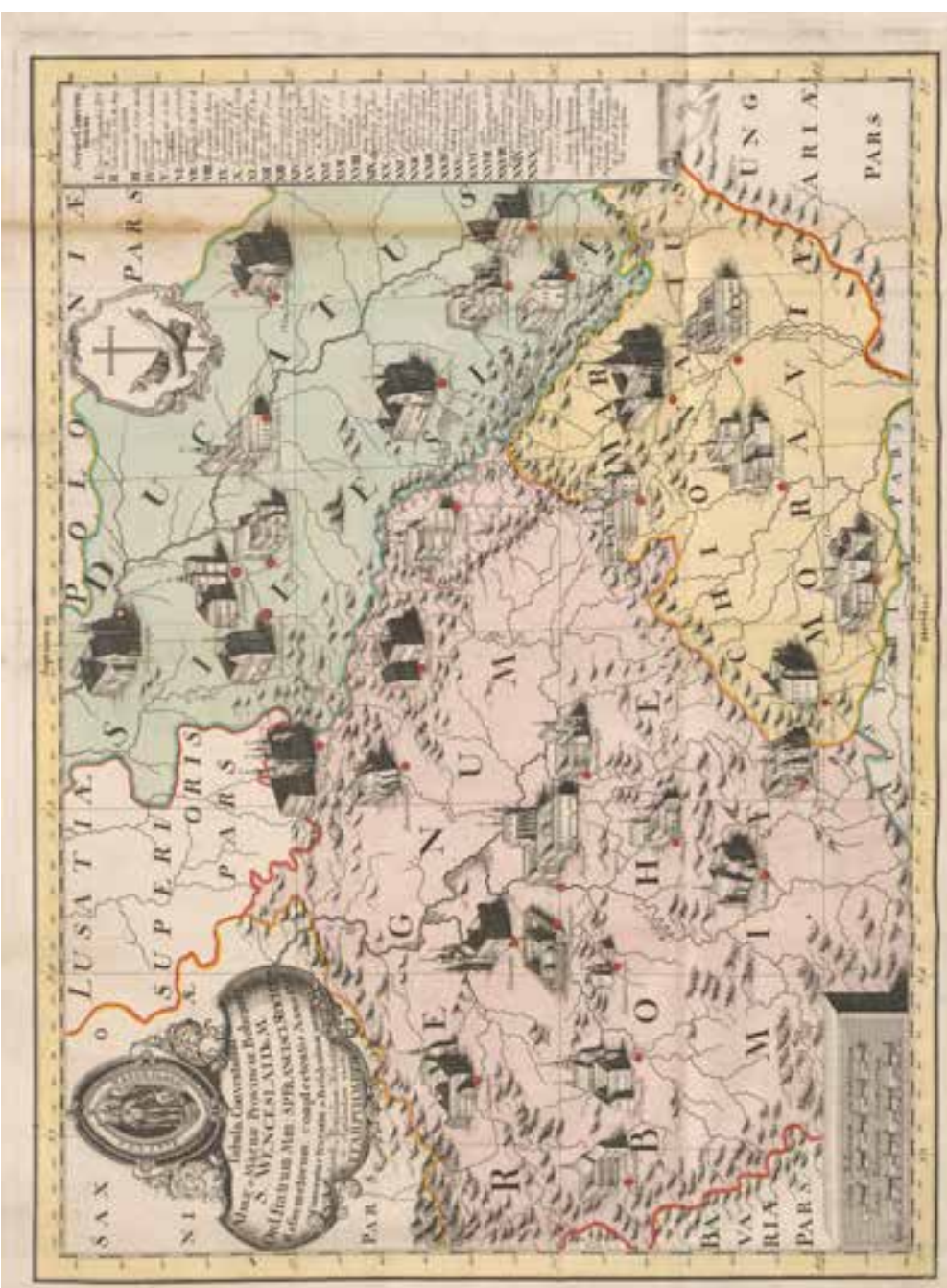

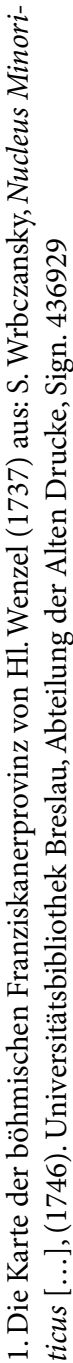




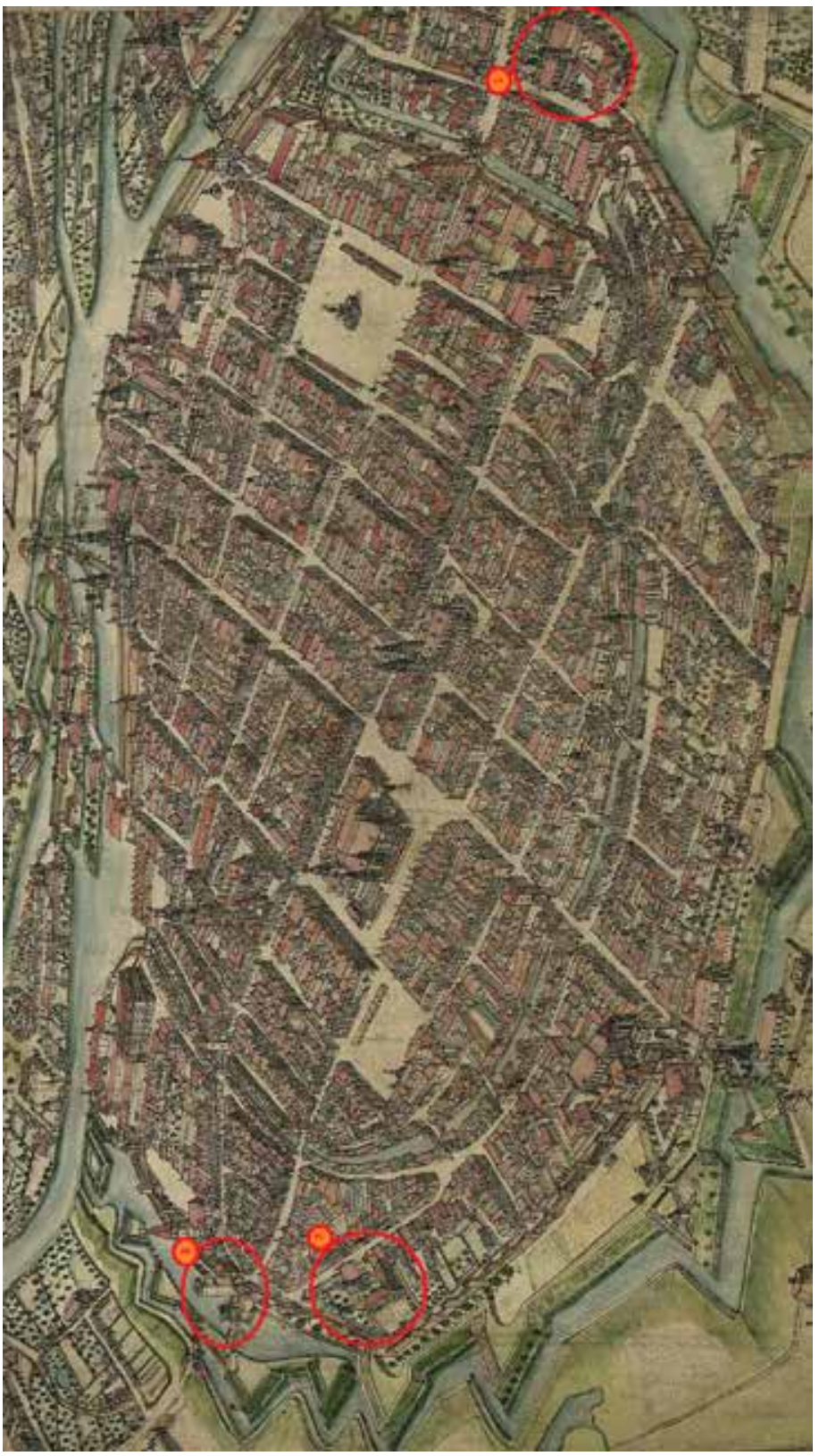

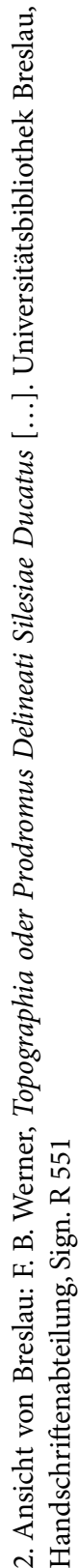




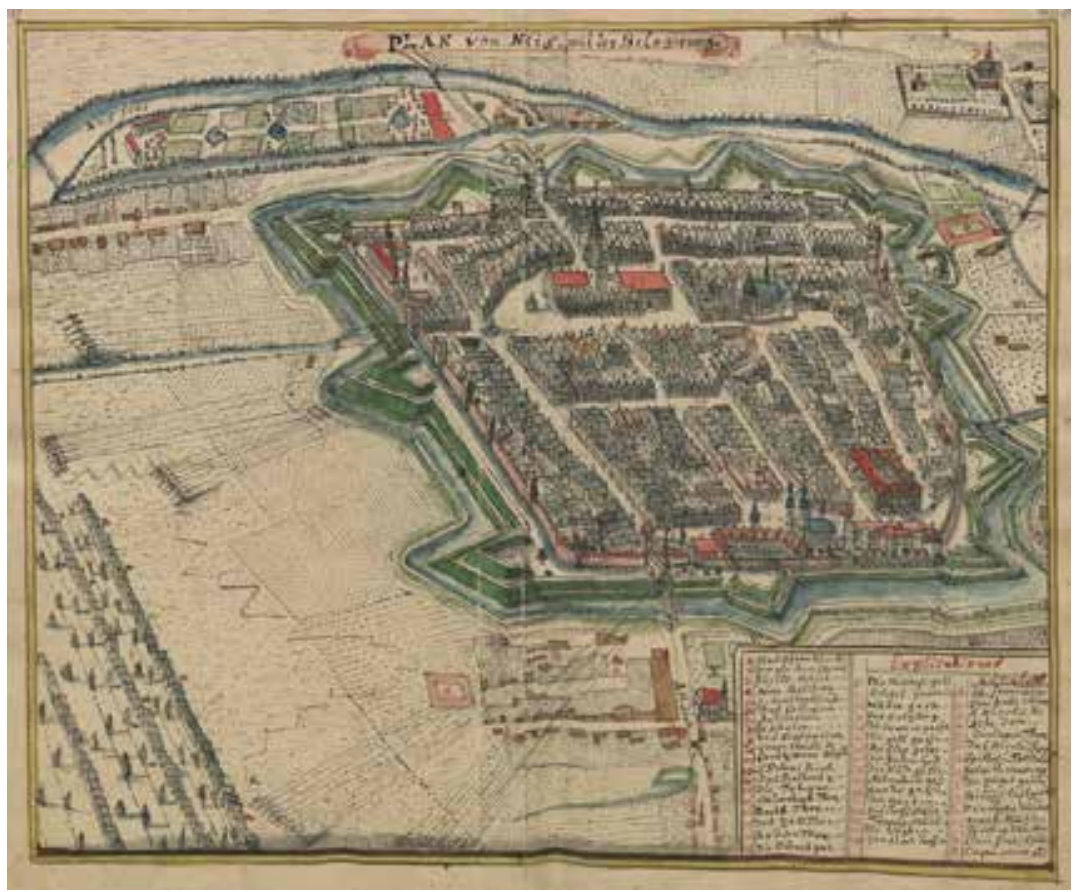

3. Ansicht von Neisse: F. B. Werner, Silesia in Compendio seu Topographia das ist Praesentatio und Beschreibung des Herzogthums Schlesiens [...], Pars I: Universitätsbibliothek Breslau, Handschriftenabteilung, Sign. IV F 113b, Vol. 1 
